\title{
In Situ Observation of Hematite Nanoparticle Aggregates by Using Liquid Cell Transmission Electron Microscopy
}

Juan Liu ${ }^{\mathrm{a},{ }^{*}}$, Zhiwei Wang ${ }^{\mathrm{b},{ }^{*}}$ Anxu Sheng ${ }^{\mathrm{a}}$, Feng Liu ${ }^{\mathrm{a}}$, Fuyu Qin ${ }^{\mathrm{b}}$, Zhong Lin Wang, ${ }^{\mathrm{b},{ }^{*}}$

* Corresponding author:

Juan Liu: juan.liu@pku.edu.cn;

Zhong Lin Wang: zlwang@gatech.edu ;

Zhiwei Wang: wangzhiwei@binn.cas.cn

7 Pages

2 Sections

4 Figures

3 Movies 


\section{Section S1:}

\section{Hematite synthesis:}

3 The HM1 sample was synthesized by slowly dripping $80 \mathrm{~mL}$ of $1 \mathrm{M}$ ferric nitrate solution at

4 the speed of $0.5 \mathrm{~mL} / \mathrm{min}$ into $1 \mathrm{~L}$ of boiling distilled MilliQ water that was stirred vigorously

5 in a HDPE bottle. After the drip solution was consumed, the resulting suspension was

6 removed from the heat quickly and cooled overnight for further cleaning process. The HM2

7 sample was synthesized by pre-heating $500 \mathrm{~mL}$ of $0.002 \mathrm{M} \mathrm{HCl}$ within a screw-cap HDPE

8 bottle at $98{ }^{\circ} \mathrm{C}$ overnight in an oven. Then, $4.04 \mathrm{~g}$ of $\mathrm{Fe}\left(\mathrm{NO}_{3}\right)_{3} \cdot 9 \mathrm{H}_{2} \mathrm{O}$ were added to the

9 solution. After the bottle was closed and vigorously shaken, it was returned to the oven immediately and held at $98{ }^{\circ} \mathrm{C}$ for 7 days. After this aging, the suspension was cooled

11 overnight before dialysis. The two synthesis suspensions were, respectively, dialyzed in

12 standard grade Cellulose dialysis membranes (1000 MWCO, Spectra Por) against MilliQ

13 water until the conductivity remained constant. The dialyzed suspension was stored in closed HDPE bottles and stored in dark at $4{ }^{\circ} \mathrm{C}$. 

non-overlapping equal boxes that would fill the projected surface area of the aggregate.

\section{Section S2}

\section{Image Analysis:}

Structural parameters of each aggregate, including $D_{m}, D_{f}$, and aspect ratio $(p)$, were measured from TEM images using a public domain, Java based image processing program ImageJ (National Institutes of Health, Bethesda, MD) and the FracLac plugin. The original TEM image was binarised by selecting brightness thresholds. The properties of the aggregates, such as area, perimeter, circularity, and the properties of the smallest rectangular area enclosing an aggregate, were derived from the projected binary images after image processing. The parameters, including $D_{m}$, aspect ratio, $R_{g}$, and $N$, were calculates from the measured values. The 2D fractal dimension $\left(D_{f}\right)$ of an aggregate was derived through box counting analysis by the FracLac plugin ${ }^{23}$. The $D_{f}$ was determined from the slope on a least square linear fit of the plot of $\log n$ versus $\log$ (box size), where $n$ is the number of 


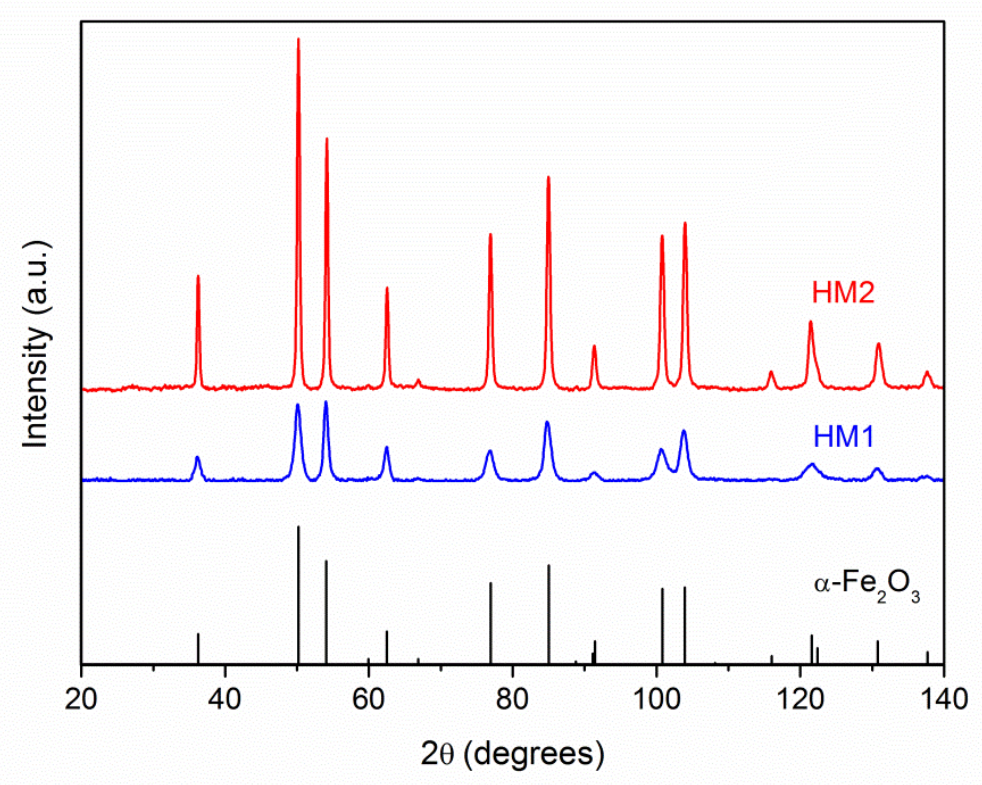

29

30 Figure S1 XRD patterns of HM1 and HM2 NPs. Only hematite phase was observed in the

31 two samples. The broader peaks of HM1 indicate its smaller particle size, which is consistent with the TEM results. 

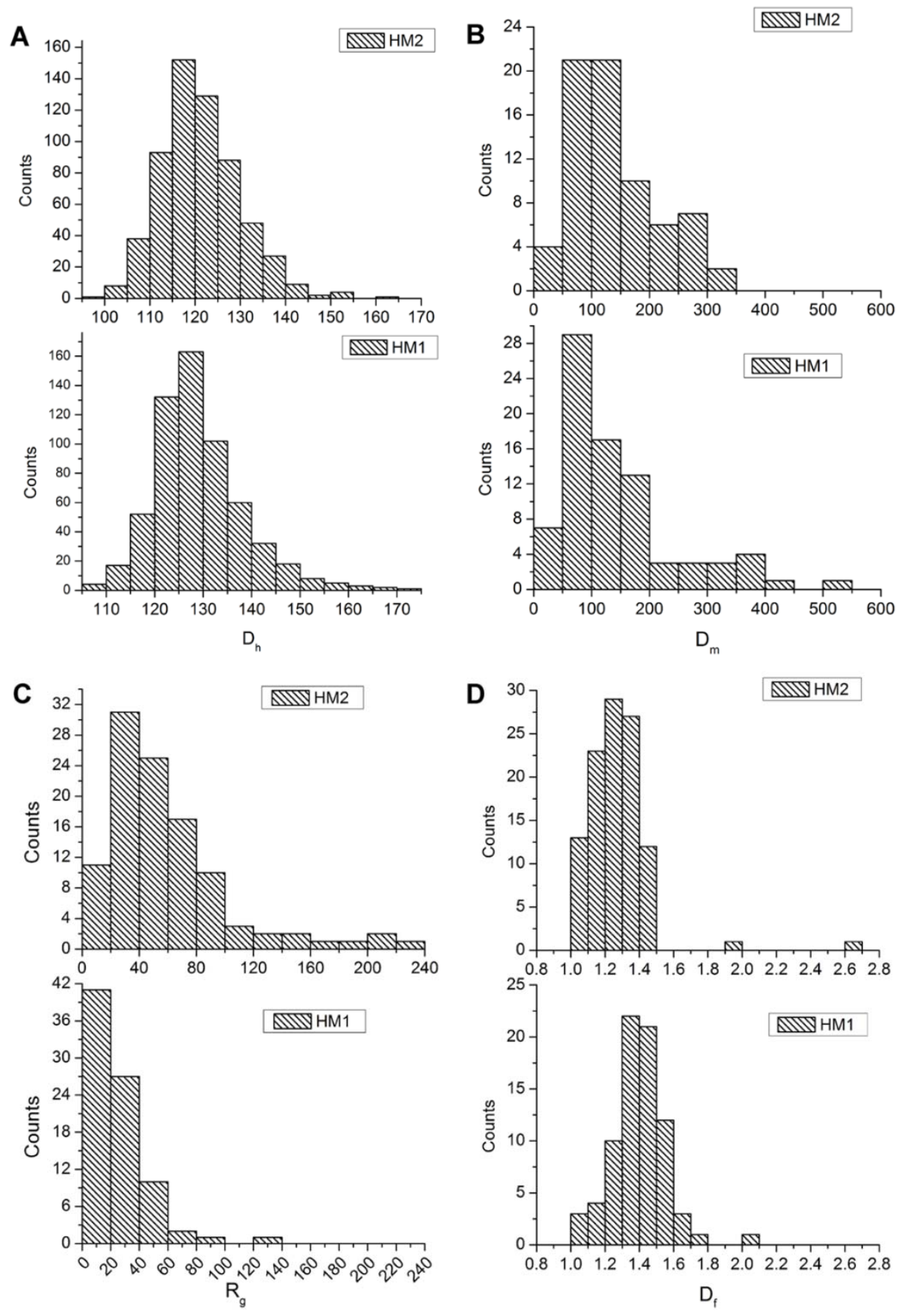

Figure S2 Distribution of hydrodynamic diameter $\left(D_{h}, \mathrm{~A}\right)$, mobility-equivalent diameter $\left(D_{m}\right.$, B), the radius of gyration $\left(R_{g}, \mathrm{C}\right)$, fractal dimension $\left(D_{f}, \mathrm{D}\right)$ of HM1 and HM2 aggregates in water. The hematite concentration in the two samples was $1.2 \mathrm{mM}$. 

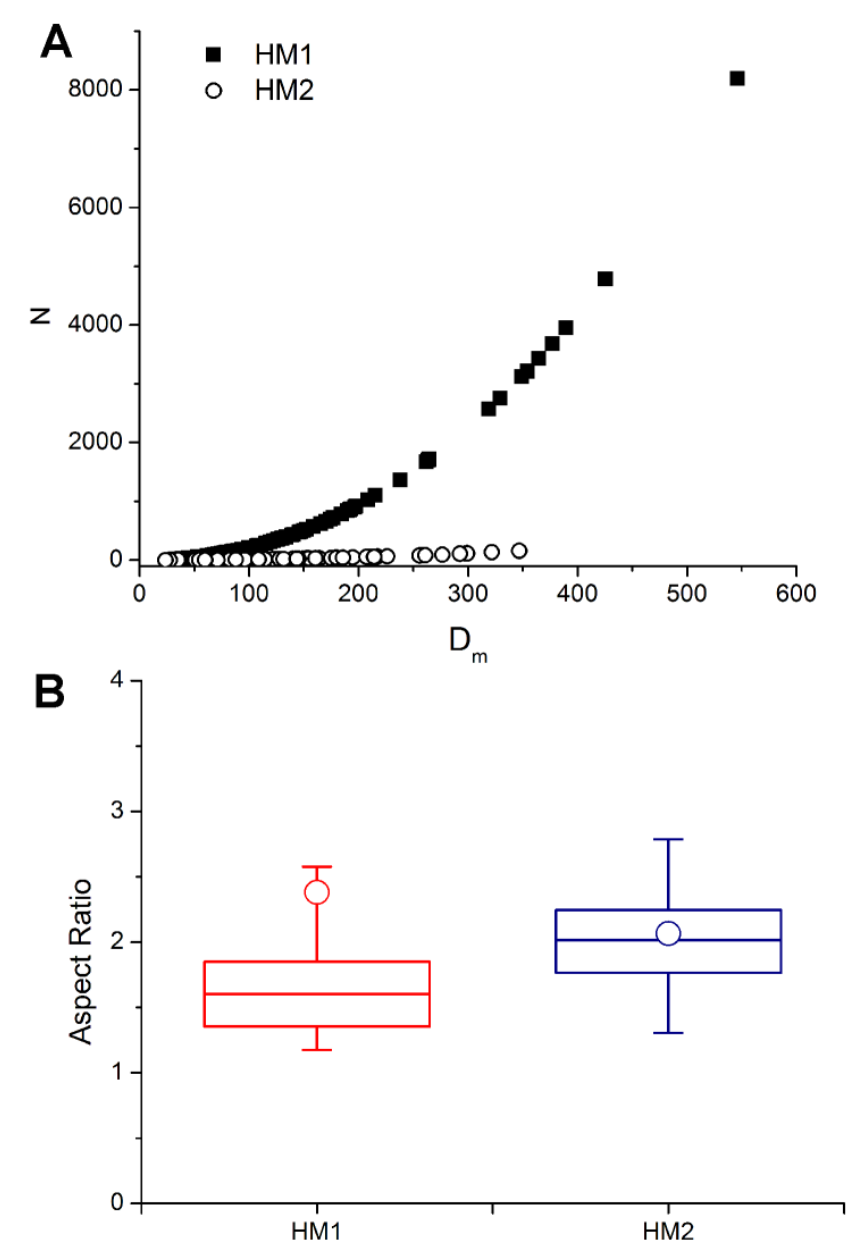

Figure S3 (A) the number of primary particles $(N)$ in a NP aggregate as a function of the aggregate's mobility-equivalent diameter $\left(D_{m}\right)$. In the aggregate with the same $D_{m}$, there are much more primary particles in HM1 than in HM2. The difference exponentially increases with increasing $D_{m}$. (B) Comparison of aspect ratios of aggregates in HM1 and HM2. The box indicates interquartile range (from $25^{\text {th }}$ to $75^{\text {th }}$ percentile) with the median indicated by the line in the middle. The circle represents the mean. The error bar indicates the range from $5^{\text {th }}$ percentile to $95^{\text {th }}$ percentile. 


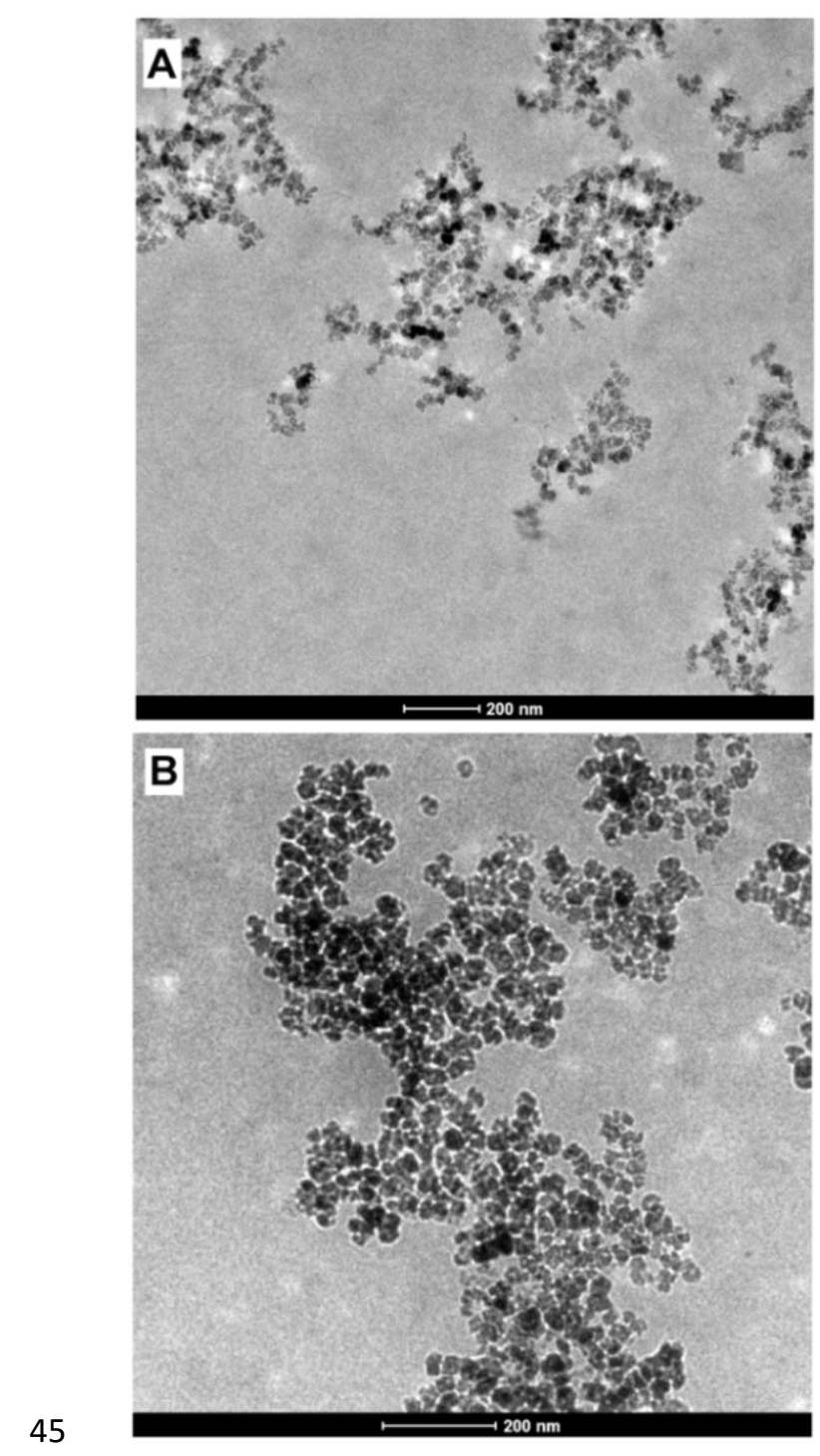

46 Figure S4 Representative TEM images of HM1 NP aggregates before and after adding the $47 \quad \mathrm{NaCl}$ solution. 
49 Movie S1 Example of HM1 aggregates moving in Milli-Q water. Movie was recorded at a

50 rate of 4 frames per second and with an estimated dose rate was 0.7 electrons/(s $\left.\AA^{2}\right)$

51 Movie S2 Example of HM2 aggregates moving in Milli-Q water. Movie was recorded at a

52 rate of 4 frames per second and with an estimated dose rate of 49 electrons/(s $\left.\AA^{2}\right)$

53 Movie S3 Example of HM1 aggregates moving in $\mathrm{NaCl}$ solution. Movie was recorded at a 54 rate of 4 frames per second and with an estimated dose rate of 22.8 electrons/(s $\left.\AA^{2}\right)$

55 